\title{
MANEJO DE LAS INYECCIONES INTRAVÍTREAS
}

\section{MANAGEMENT OF INTRAVITREAL INJECTIONS}

\author{
GÓMEZ-ULLA F ${ }^{1,3}$, BASAURI E ${ }^{2,4}$, ARIAS L ${ }^{1,5}$, MARTÍNEZ-SANZ F F,6
}

\begin{abstract}
RESUMEN
Objetivo: La inyección intravítrea es una técnica mínimanente invasiva de demostrada eficacia en el tratamiento de numerosas enfermedades vitreorretinianas. Su uso, gracias a nuevas medicaciones e indicaciones, se ha generalizado en los últimos años. La práctica de esta técnica sin las precauciones adecuadas, puede causar complicaciones graves, en especial la infección.

La finalidad de esta Guía es difundir entre los oftalmólogos un compendio de recomendaciones estandarizadas que faciliten la administración de inyecciones intravítreas, con las máximas medidas de seguridad y fiabilidad.
\end{abstract}

Método: Se efectúa:

1. Una revisión bibliográfica de la evidencia científica publicada hasta la fecha.

2. Consulta de práctica clínica habitual entre especialistas con experiencia acreditada.

3. Contraste de nuestra información con la contenida en las guías publicadas en otros países.

Resultado: Aunque no hay un consenso absoluto, las maniobras recomendadas en esta Guía parecen, hasta el día de la fecha, las más adecuadas para minimizar los riesgos de complicaciones, en espe-

\begin{abstract}
Purpose: The intravitreal injection is a minimally invasive technique which has been proved to be an effective therapy in the management of numerous vitreoretinal diseases. Its use has been rapidly increased in the last few years due to new medications and indications. However, non-standardized performance of this procedure might cause severe complications, being of special concern intraocular infection.

The aim of this Guide is to give ophthalmologists, with the information we have up to date, the guidelines needed to make this procedure safe.

Methods: 1. Reviewing the literature regarding evidence published up to date.

2. Consulting with experienced surgeons their common practice in this tecnique.

3. Contrasting the information from this Guide with those guides published in other countries.

Results: Although there is no complete consensus regarding the intravitreal injection procedure technique, the recomendations in this Guide are, up to date from our point of view, the most appropriate to prevent complications, specially infection. Of most importance are antisepsis with povidone iodine, the
\end{abstract}

Recibido: 14/7/09. Aceptado: 20/8/09.

Sociedad Española de Retina y Vítreo.

1 Doctor en Medicina.

${ }^{2}$ Licenciado en Medicina.

${ }^{3}$ Complejo Hospitalario Universitario de Santiago de Compostela. Instituto Tecnológico de Oftalmología (ITO). España.

${ }^{4}$ Instituto Balear de Oftalmología. Hospital Son Llàtzer (Palma de Mallorca). España.

${ }^{5}$ Hospital Universitari de Bellvitge. Institut de la Màcula i de la Retina. Centro Médico Teknon (Barcelona). España.

${ }^{6}$ Hospital Universitario Marqués de Valdecilla (Santander). España.

Este trabajo forma parte de la colección de Guías Práctica Clínica de la Sociedad Española de Retina y Vítreo y ha sido validada previamente por los siguientes revisores: Fernando Arévalo (Venezuela), Borja Corcóstegui (España), Víctor González (EE.UU.), Antonio Piñeiro (España).

Correspondencia:

Francisco Gómez-Ulla

Hospital Provincial de Conxo

Rúa Ramón Baltar, s/n

15705 Santiago de Compostela

España

E-mail: gomezulla@itogalicia.es 
cial la infección. Se consideran de particular importancia la antisepsis con povidona yodada, el uso de guantes y materiales estériles y una técnica adecuada de inyección.

Conclusiones: Series muy grandes de inyecciones intravitreas realizadas en consulta y aplicando las medidas aquí recomendadas, presentan una incidencia muy baja de complicaciones, en especial la sospecha de infección intraocular.

Palabras clave: Inyección intravítrea, endoftalmitis, anti-VEGF, triamcinolona. use of sterile material and gloves and an adecuate injection technique.

Conclussions: Large series published of intravitreal injections which took place in clinic settings applying a procedure tecnique as recomended in this Guide, presented a very low incidente of complications, specially endophthalmitis (Arch Soc Esp Oftalmol 2009; 84: 377-388).

Key words: Intravitreal injection, endophthalmitis, ant-VEGF, triamcinolone.

\section{INTRODUCCIÓN}

La primera referencia al uso de la inyección intravítrea (IIV) fue realizada en 1911 por Ohm, quien la utilizó como técnica de introducción de aire en el ojo para reparar el desprendimiento de retina (1).

A partir de 1945 se utiliza la IIV como vía de administración de fármacos con la introducción de penicilina para el tratamiento de la endoftalmitis $(2,3)$ continuándose con el uso de este procedimiento en el tratamiento del desprendimiento de retina (4-7), de las endoftalmitis $(8,9)$ y de la retinitis por CMV $(10,11)$.

En estos últimos años se ha producido un aumento exponencial de su uso por la expansión de sus aplicaciones clínicas, con la inyección de acetonido de triamcinolona para el edema macular, alteraciones vasculares retinianas, inflamaciones intraoculares, neovascularización coroidea (12-20) primero, y mas adelante con el desarrollo de fármacos antiangiogénicos: pegaptanib sódico (21-24), ranibizumab (25-27) y bevacizumab (28-32) para el tratamiento de la neovascularización coroidea y otras patologías (obstrucciones de rama y de vena central de la retina, edema macular diabético, retinopatía diabética proliferativa, edema macular quístico y glaucoma neovascular).

Aunque algunos de estos fármacos no estén diseñados para su uso intraocular, esta vía de administración es unánimemente aceptada, siendo en la actualidad su uso generalizado, reconociéndose sus ventajas frente a otras vías de administración en el tratamiento de ciertas enfermedades oculares.

Estamos aplicando estos tratamientos a un gran número de pacientes, y así se prevé que continuare- mos haciéndolo, hasta que encontremos otras vías tanto o más eficaces para la introducción de fármacos dentro del globo ocular. Por otro lado, estamos efectuando las inyecciones de forma repetida, en tanto no hallemos la manera de mantener la medicación dentro del ojo o su efecto terapéutico dure períodos más largos de tiempo.

Debemos tener en cuenta que el resultado de estos tratamientos mediante IIV va a depender no sólo de la eficacia de la medicación inyectada, sino también de sus riesgos (seguridad, tolerancia y complicaciones propias del fármaco). Distintos fármacos presentan riesgos distintos y en proporciones diferentes. A estos hay que añadir los riesgos propios del procedimiento.

La complicación más temida de este procedimiento por ser potencialmente devastadora, es la endoftalmitis; no obstante, tomando ciertas medidas de asepsia que debemos protocolizar, el riesgo es bajo.

A pesar de carecer de estudios que comparen entre sí ciertas maniobras de prevención, en esta guía nos ceñiremos a unas recomendaciones que, o bien ya están demostradas como útiles en la prevención de infección en la IIV, o se consideran medidas universales de asepsia que podemos aplicar a este procedimiento.

\section{RIESGOS DE LA INYECCIÓN INTRAVÍTREA}

En el año 2004 Jaeger y cols publican la revisión más importante que se ha llevado a cabo sobre los riesgos de este procedimiento (33), en un número de 14.866 inyecciones intravítreas de: agentes antivirales (ganciclovir, cidofovir, fomivirsen), 
triamcinolona, fármacos anti-VGF, gas, TPA, y metotrexate.

Se describen distintos tipos de riesgos: endoftalmitis, incluyendo casos de pseudoendoftalmitis $(0,3 \%)$, desprendimiento de retina $(0,9 \%)$, hemorragia intraocular $(1,3 \%)$, catarata (desarrollo o progreso) $(9,9 \%)$, (porcentaje que aumenta cuando el seguimiento es más largo), uveítis/iritis (6,3\%), hipertensión ocular mantenida (2,4\%-38,3\%); más raramente hipotensión ocular, atrofia óptica, catarata traumática y obstrucción vascular retiniana. La hipertensión ocular mantenida, la uveítis y la aparición o progresión de una catarata, estarían relacionados con el fármaco introducido, al igual que la uveítis, la hipotonía ocular y la obstrucción vascular retiniana (cidofovir y fomivirsen). El desprendimiento de retina estaría relacionado con la patología ocular subyacente (retinopatía por CMV y hemovítreo diabético).

Este estudio considera la endoftalmitis infecciosa como el riesgo más importante del procedimiento, siendo su probabilidad de $0,3 \%$ si se incluyen los casos de pseudoendoftalmitis y endoftalmitis con cultivo negativo. Queda reducida al $0,2 \%$ en casos de endoftalmitis con cultivo positivo. La incidencia es de $0,1 \%$ si se excluyen los casos por inyección de triamcinolona intravítrea.

En noviembre de 2005 el Comité Etico del Centro Médico del Pacifíco en California (34), propuso un cuestionario clínico para la recogida de datos de seguridad (disponible en la red) tras la inyección intravítrea de bevacizumab: «The International Intravitreal Bevacizumab Safety Survey».

Desde noviembre de 2005 a junio de 2006 se recogieron datos de 70 centros de 12 países con un total de 7113 inyecciones en 5228 pacientes, del que se desprendieron las siguientes conclusiones:

- En relación con el procedimiento se describieron las siguientes complicaciones: abrasiones corneales $(0,15 \%)$, daños al cristalino $(0,01 \%)$, endoftalmitis $(0,014 \%)$, desprendimientos de retina $(0,04 \%)$ y hemorragias subconjuntivales $(0,03 \%)$.

- En relación con el fármaco se comunicaron los siguientes efectos adversos: inflamación $(0,14 \%)$, progresión de cataratas $(0,01 \%)$, oclusión de la arteria central de la retina $(0,01 \%)$, hemorragia subretiniana $(0,06 \%)$ y desprendimiento del epitelio pigmentario $(0,06 \%)$. A nivel sistémico se halló un 0,21\% de casos de HTA, 0,01\% de accidentes vasculares transitorios y también un $0,01 \%$ de trombosis venosas profundas.

En enero de 2008, Wu y cols. (35) publican un estudio retrospectivo y colaborativo del PACORES sobre la seguridad y complicaciones de las inyecciones intravítreas de $1,25 \mathrm{mg}$ o 2,5 mg de bevacizumab con un seguimiento de 12 meses. Recogen 4303 IIV en 1.310 ojos de 1.173 pacientes. Comunican complicaciones sistémicas en el 1,5\% de los pacientes $(0,59 \%$ de elevación de la tensión arterial, $0,5 \%$ de accidentes cerebro-vasculares, $0,4 \%$ de infartos de miocardio, $0,17 \%$ de aneurismas arteriales ilíacos y $0,4 \%$ de muertes) y las complicaciones oculares incluyeron $0,16 \%$ de endoftalmitis bacteriana, $0,16 \%$ de desprendimientos de retina traccional, $0,09 \%$ de uveitis, $0,02 \%$ de desprendimientos de retina regmatógeno y $0,02 \%$ de hemorragias vítreas).

Por otra parte, en marzo de ese mismo año, Moshfeghi (36) publica una revisión de 19.830 inyecciones intravítreas de fármacos anti-VEGF realizadas en el Bascom Palmer Eye Institute de Miami, entre enero de 2005 y noviembre de 2007, con un resultado del $0,015 \%$ de endoftalmitis. Y en mayo, Pilli y cols (37) publican una revisión de 10.254 inyecciones intravítreas de medicación antiVEGF (406 de pegaptanib sódico, 6.347 de ranibizumab y 3.501 de bevacizumab) en el que se presenta una incidencia de sospecha de endoftalmitis de $0,029 \%$.

En estudios anteriores con inyección intravítrea de fármacos antiangiogénicos, la incidencia de endoftalmitis por inyección es de $0,1 \%$ a $0,2 \%$ $(23,38,39)$.

Los diversos estudios presentan un riesgo de endoftalmitis infecciosa más alto por inyección de triamcinolona: entre el 0,1\% (40), 0,2\% (33), el $0,87 \%$ (41) y el $1,9 \%$ (42). A esto hay que añadir los casos de endoftalmitis no infecciosa y pseudoendofalmitis por cristales de triamcinolona en cámara anterior: entre el $0,1 \%$ (33) y el $0,8 \%(43,44)$.

\section{NECESIDAD DE PROTOCOLIZAR EL PROCEDIMIENTO}

Como sabemos, la endoftalmitis es la complicación más temida de este procedimiento por sus posibles consecuencias, pero su riesgo es bajo 
tomando ciertas medidas de asepsia, por lo que proponemos aquí aplicar unas medidas que, o bien ya están demostradas como útiles en la prevención de la infección en la inyección intravítrea, o se consideran pautas universales de asepsia aplicables a esta técnica.

En los estudio multicéntricos, prospectivos y randomizados de Pegaptanib (23) hubo una reducción significativa de incidencia de la infección después de cambiar el protocolo de la IIV. 8 de los 12 casos de endoftalmitis se produjeron en el primer año del estudio, y una vez modificado el protocolo, no hubo más casos de endoftalmitis. Esto nos permite considerar que la técnica de inyección es muy importante a la hora de prevenir la endoftalmitis.

Se han publicado guías con recomendaciones para la IIV. La primera, ya mencionada, data del año 2004 después de una reunión de expertos y fue publicada en la revista «Retina» (45). Tanto la Sociedad Alemana de Oftalmología en el año 2005 (46), como el Colegio Británico de Oftalmólogos en el 2006 (47), editaron sus propias recomendaciones para éste procedimiento.

\section{REQUISITOS ANTES DE LA INYECCIÓN}

\section{Explicar al paciente:}

a) Cuál es el objetivo del tratamiento.

b) Cómo es el procedimiento y tranquilizarle.

c) Cuáles son sus expectativas y posibles riesgos.

d) La posibilidad de inyecciones repetidas y su frecuencia en el tiempo.

e) Alternativas de tratamiento si las hubiese.

f) Razonamiento para utilizar un fármaco no aprobado para su uso intraocular o aprobado para otra enfermedad, si es el caso.

2. Firma del consentimiento informado del procedimiento, una vez que el paciente ha entendido lo anteriormente explicado.

3. Solicitud de medicación de uso compasivo, o en su caso, aplicación de la legislación vigente, si se utiliza un fármaco no aprobado para su uso intraocular.

\section{PROFILAXIS ANTES DE LA INYECCIÓN}

\section{Tratar previamente la infección ocular externa}

La infección ocular externa (incluyendo la blefaritis activa), palpebral o lagrimal, deberá ser tratada antes de realizar el procedimiento.

Las bacterias de la superficie ocular son la fuente más común de microorganismos causantes de la endoftalmitis postquirúrgica. También se debe considerar que anomalías palpebrales como el ectropion, pueden constituir riesgo de endoftalmitis post quirúrgica (48-51).

\section{Utilización de todos los colirios estériles}

Se recomienda el uso de colirios estériles, tanto para la dilatación pupilar como para la anestesia tópica, ya que está demostrada la contaminación de los colirios oftálmicos de uso múltiple en las consultas de oftalmología (52).

\section{LUGAR DE LA INYECCIÓN Y PROFILAXIS DURANTE EL PROCEDIMIENTO}

\section{Inyección en consulta, sala de curas o quirófano}

No hay una recomendación específica sobre el lugar requerido para realizar el procedimiento (consulta, sala de curas o quirófano), siempre y cuando ese espacio presente la suficiente comodidad, tanto para el paciente como para el oftalmólogo, y así mismo permita la realización de una técnica estéril $(37,45)$.

\section{Uso de material estéril}

Para la realización de la técnica estéril, se precisan precauciones universales, como son el uso por parte del cirujano de guantes y materiales estériles (dentro de estos últimos):

- Blefarostato estéril (que evitará el contacto de la aguja con párpados y pestañas).

- Calibrador estéril. 
- Aguja estéril de 30 ó 32 G.

- Pinzas, bastoncillos de algodón o hemostetas también estériles.

\section{Uso de anestesia tópica}

La experiencia nos demuestra que el colirio anestésico tópico proporciona un procedimiento cómodo para el paciente. Puede valorarse la utilización de anestesia subconjuntival, pero requiere una manipulación asociada y la posible formación de una hemorragia subconjuntival.

En cuanto al gel de lidocaína, un estudio del año 2005 refiere que éste procura comodidad para la realización de las IIV, y causa menos hemorragia subconjuntival que la inyección subconjuntival de anestesia (53). Pero sin embargo, otro estudio de este mismo año, relaciona el uso de este gel como un posible factor de riesgo de infección después de la cirugía de cataratas (54). También el gel de lidocaína, puede actuar como barrera para la acción de la povidona yodada en la superficie ocular, limitando su acción bactericida. Y además un gel que no esté preparado como producto estéril, puede ser él mismo una fuente de contaminación. Por todo ello, no recomendamos su uso.

\section{Reducción de la flora bacteriana de la superficie ocular y de los párpados}

Uso de povidona yodada al 5 y $10 \%$ respectivamente.

Existen varias maneras de reducir la flora bacteriana de la superficie ocular: el uso de la povidona yodada y la higiene palpebral, asociados al uso de antibiótico tópico previo al procedimiento y al aislamiento estéril del campo quirúrgico:

a) El uso de la povidona yodada tópica al $5 \%$ en saco conjuntival (55), es el único método que ha demostrado reducir el riesgo de endoftalmitis postoperatoria en un estudio prospectivo de cirugía de cataratas (56).

A diferencia de la aplicación de dos gotas de povidona yodada al $5 \%$ sobre el saco conjuntival, el lavado conjuntival con $10 \mathrm{cc}$ del producto, está asociado a un menor crecimiento de cultivo bacteriano de muestras conjuntivales tomadas durante la cirugía de catarata (57). Se desconoce si esta diferencia tiene algún efecto en la prevención de endoftalmitis.

Se recomienda la limpieza externa de los párpados con povidona yodada al $10 \%$ y conjuntival al $5 \%(45,55)$.

El riesgo de alergia a la povidona yodada es muy bajo y se recomienda realizar pruebas en la piel para confirmar dicha alergia, que no se debe confundir con irritación (una alergia a un contraste yodado no supone necesariamente alergia a la povidona yodada).

b) No hay evidencia de que el uso de antibiótico tópico previo al procedimiento disminuya el riesgo de endoftalmitis.

Hay un estudio que presenta una acción sinérgica entre la povidona yodada y el antibiótico tópico en la reducción de la flora bacteriana de la superficie ocular $(58,59)$. Otros estudios demuestran cómo el colirio antibiótico aplicado a la conjuntiva, produce una marcada reducción de la flora bacteriana en la superficie del ojo (60-62).

Pero, como hemos apuntado, no hay evidencia de que todo lo anteriormente expuesto, de cómo resultado una reducción del riesgo de endoftalmitis posquirúrgica.

Por otra parte, Deramo y cols. (63), presentan una serie de 42 ojos en 42 pacientes con endoftalmitis postquirúrgica aguda, y todos ellos fueron tratados con antibiótico de amplio espectro preoperatoriamente. Esto indica que, aunque se utilice el antibiótico antes del procedimiento, la endoftalmitis puede producirse.

Otro estudio muestra cómo se crean resistencias a antibióticos de amplio espectro utilizados comúnmente como profilácticos en la cirugía intraocular (64).

c) Entendemos que el uso de un campo quirúrgico estéril no es estrictamente necesario, puesto que la simplicidad y rapidez de la técnica implican poco riesgo de contaminación desde el «exterior «del ojo y anejos.

\section{Evitar la manipulación palpebral excesiva}

La manipulación palpebral excesiva se ha descrito como causa de aumento de la flora bacteriana en conjuntiva (65). Un blefarostato que comprima demasiado las pestañas puede producir el mismo efecto.

Se recomienda evitar ambas situaciones. 


\section{Paracentesis}

Se recomienda evitar siempre que sea posible abrir una nueva vía de entrada al ojo, como es la realización de una paracentesis (45).

\section{Mascarilla}

No hay datos en nuestra literatura que sugieran que el uso de una mascarilla por parte del cirujano pueda tener un efecto profiláctico, ni que su ausencia aumente el riesgo de infección en este procedimiento.

\section{Antibiótico tópico de amplio espectro post- inyección}

La inyección intraocular abre una puerta de entrada a microorganismos al espacio subconjuntival, por lo que se recomienda el uso del antibiótico tópico de amplio espectro al final del procedimiento.

\section{SECUENCIA DEL PROCEDIMIENTO}

\section{Dilatación pupilar para la visualización del fondo de ojo}

Aunque no es imprescindible, creemos que es muy aconsejable, tanto para una adecuada visualización posterior del fondo de ojo, como para el control del «latido venoso»y de la «palidez» papilar, por si fuese necesaria alguna actuación posterior.

\section{Anestesia tópica con colirio estéril}

Ya sea lidocaina $2 \%$ en ampolla esteril, o colirio de anestesico (tetracaina) abierto en ese momento.

\section{Limpieza con povidona yodada}

Emplearemos, como ya se ha comentado, povidona yodada al $10 \%$ para la piel de los parpados, borde palpebral y pestañas, y al 5\% para el saco conjuntival, dejándola actuar durante tres minutos.

\section{Aplicación del blefarostato}

Creemos más apropiados aquellos que tienen una pletina, pues separan mejor las pestañas, a la vez que aíslan el ojo del borde palpebral y de la secreción de las glándulas de Meibomio.

\section{Medición de la distancia adecuada, desde limbo hacia pars plana}

Mediremos con un compás o herramienta similar, una distancia normal desde el limbo y hacia pars-plana, de 3,5 mm en ojos afáquicos o pseudofáquicos y de $4 \mathrm{~mm}$ en ojos fáquicos.

\section{Indicar al paciente la posición del globo}

Solos o ayudados por una auxiliar que sujete ligeramente la posición de la cabeza, indicaremos al paciente que mire hacia arriba y al lado contrario al punto de la inyección, la que aplicaremos frecuentemente en el cuadrante temporal inferior.

(Algunos autores recomiendan la inyección en los cuadrantes inferiores como prevención de un posible fenómeno de Bells durante el procedimiento. También se recomiendan distintos lugares para inyectar en subsiguientes procedimientos, y evitar las 3 y 9 horas por la localización de las arterias ciliares).

\section{Movilización de la conjuntiva en el punto a inyectar con pinza o bastoncillo de algodón}

A los efectos de impedir la coincidencia del orificio conjuntival y escleral, trasladaremos unos milímetros la conjuntiva con un bastoncillo de algodón estéril, con una pinza o con el mismo instrumento que utilizamos en la medición del punto de infiltración, con el máximo cuidado para impedir una hemorragia subconjuntival. Más aún, en paciente anticoagulados.

\section{Inserción de la aguja}

Perpendicularmente a través de la esclera con la punta apuntando hacia el centro del globo para evitar dañar el cristalino. Hay que tener especial 
cuidado en no contaminar la aguja por contacto. Inyección del producto $(0,05-0,1 \mathrm{ml}$ según el fármaco) de manera suave para evitar un efecto difusor.

\section{Extracción suave de la aguja}

Se puede utilizar un bastoncillo de algodón estéril o el mismo instrumento de medición por el lado opuesto, para prevenir el reflujo del fármaco o vítreo acuoso, y el sangrado posterior.

\section{Administración de un colirio de antibiótico de amplio espectro}

Emplearemos un colirio de amplio espectro, dos gotas tras la aplicación de la infiltración y durante unos días después del procedimiento. Por ejemplo, nosotros empleamos como posología habitual, ofloxacino o ciprofloxacino, 2 gotas cada 8 horas entre 3 y 5 días posteriores a la inyección.

\section{Exploración de la percepción luminosa y visión de objetos}

Caso de ser necesario valorar si hay perfusión de la arteria centra de la retina, se puede hacer con oftalmoscopio indirecto o con lámpara de hendidura y lente de no contacto.

\section{PAUTAS POSTOPERATORIAS}

1. No existen estudios que comparen diferentes estrategias de seguimiento después del procedimiento.

2. Si se estima necesario, se puede medir la presión intraocular en los 30 minutos posteriores a la inyección (preferiblemente con tonómetro de no contacto).

3. Aunque no esté demostrada su eficacia, y a pesar de que podría aumentar el riesgo de crear resistencias bacterianas, se recomienda el uso de antibiótico tópico de amplio espectro durante los días posteriores al procedimiento pensando en la vía de entrada al espacio subconjuntival recién creada por la inyección.
4. Habitualmente no es necesario tapar el ojo y el paciente puede marcharse a su domicilio al cabo de pocos minutos.

La siguiente visita de control dependerá de la enfermedad, del fármaco inyectado y del número de la infiltración, por lo que será indicada individualmente a cada paciente.

5. Se recomienda instruir al paciente sobre los posibles efectos adversos locales y sistémicos, así como entregarle por escrito los síntomas de alarma que pueden requerir una asistencia o consulta telefónica urgente. En este escrito debe figurar en lugar destacado el principio activo del fármaco que ha sido administrado. El paciente deberá conservar esta documentación escrita durante todo el tiempo que dure su proceso ocular.

6. Normas escritas que se entregarán al paciente:

a) Fármaco intravítreo inyectado (principio activo y nombre comercial)

b) Ojo inyectado

c) Dosificación y nombre del colirio antibiótico a instilar.

d) Síntomas de alarma que requieren contactar con un oftalmólogo:

Disminución de la visión, dolor ocular a la luz, enrojecimiento del ojo diferente al causado por la inyección o secreción purulenta del ojo. Especialmente la pérdida muy importante de visión, con dolor ocular y enrojecimiento pueden ser síntomas de una endoftalmitis.

El paciente ha recibido instrucciones de no frotarse los ojos, ni sumergirse, ni que entren líquidos en su ojo durante 3 días.

Debe cumplir las instrucciones de medicaciones así como las visitas de seguimiento postoperatorias.

e) Sintomas de alarma que requieren contactar con su médico internista o acudir a un Servicio de Urgencias (especialmente con la administración de anti-VEGF):

Aunque la posibilidad de que se produzcan complicaciones severas que afecten al resto del organismo son muy bajas, deberá contactar en caso de que sienta dolor abdominal con vómitos y estreñimiento, sangrado anormal, dolor en el pecho, dificultades al hablar, dolor de cabeza importante, o debilidad de alguna extremidad. En cuanto le sea posible notificará estos acontecimientos a su oftalmólogo.

f) Teléfono de contacto de la clínica en que se ha realizado la inyección y nombre del oftalmólogo que la ha dispensado. 


\section{ALGORITMO DE INYECCIÓN INTRAVÍTREA}

\section{SECUEN CIA IN YECCION INTRAVITREA}
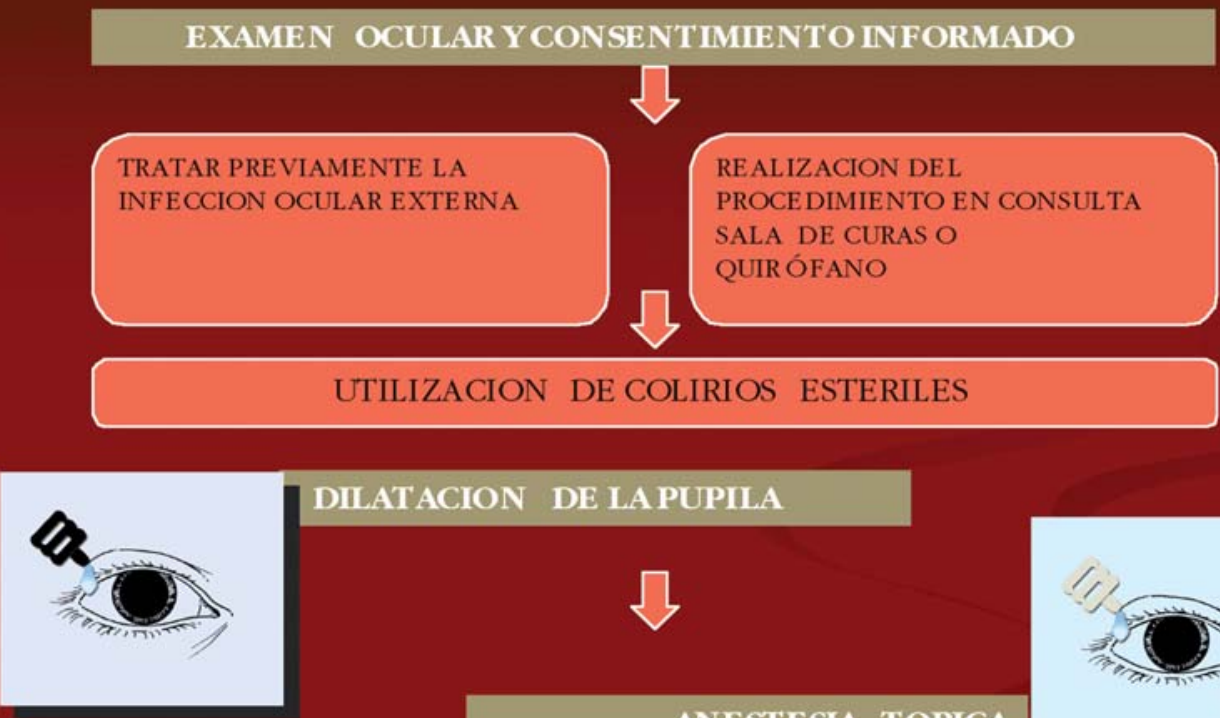

\section{DILATACION DE LA PUPILA}

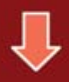

ANESTESTA TOPICA
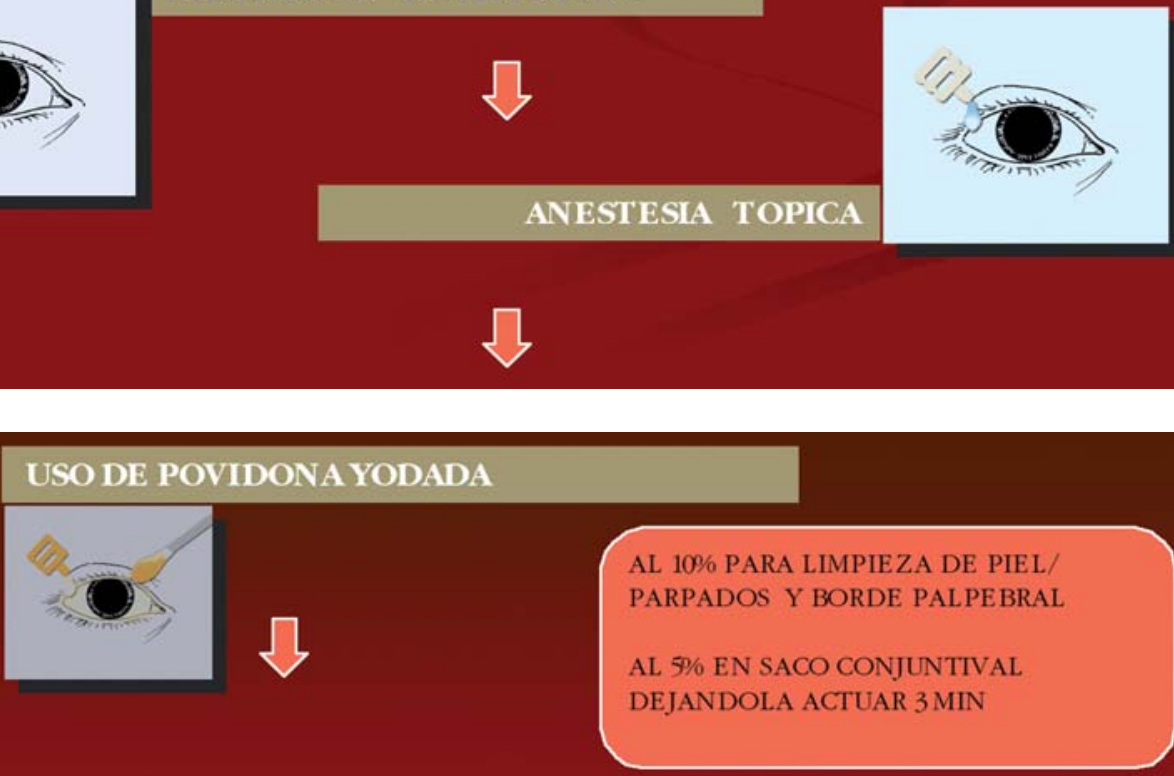

\section{USO DE GUANT ES Y MAT ERIAL ESTERILES}

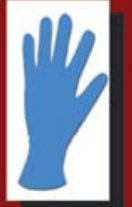

\section{BLEFAROSTATO}

CALIBRADOR

AGUJA DE 30 Ó $32 \mathrm{G}$

PINZAS/BASTONCILLOS O

HEMOSTETAS

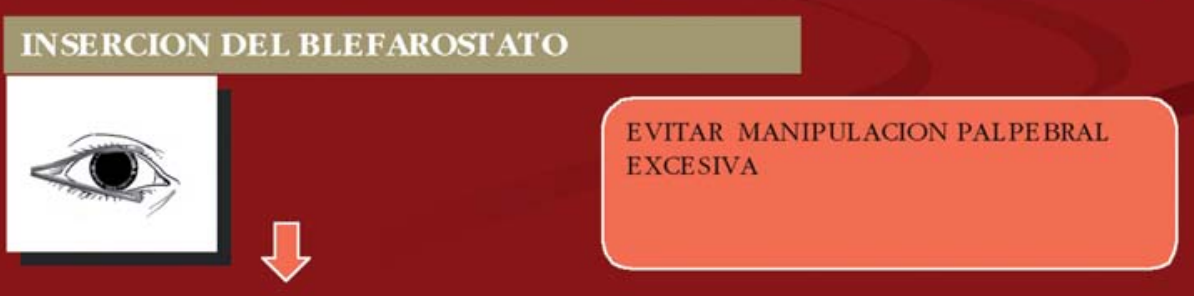



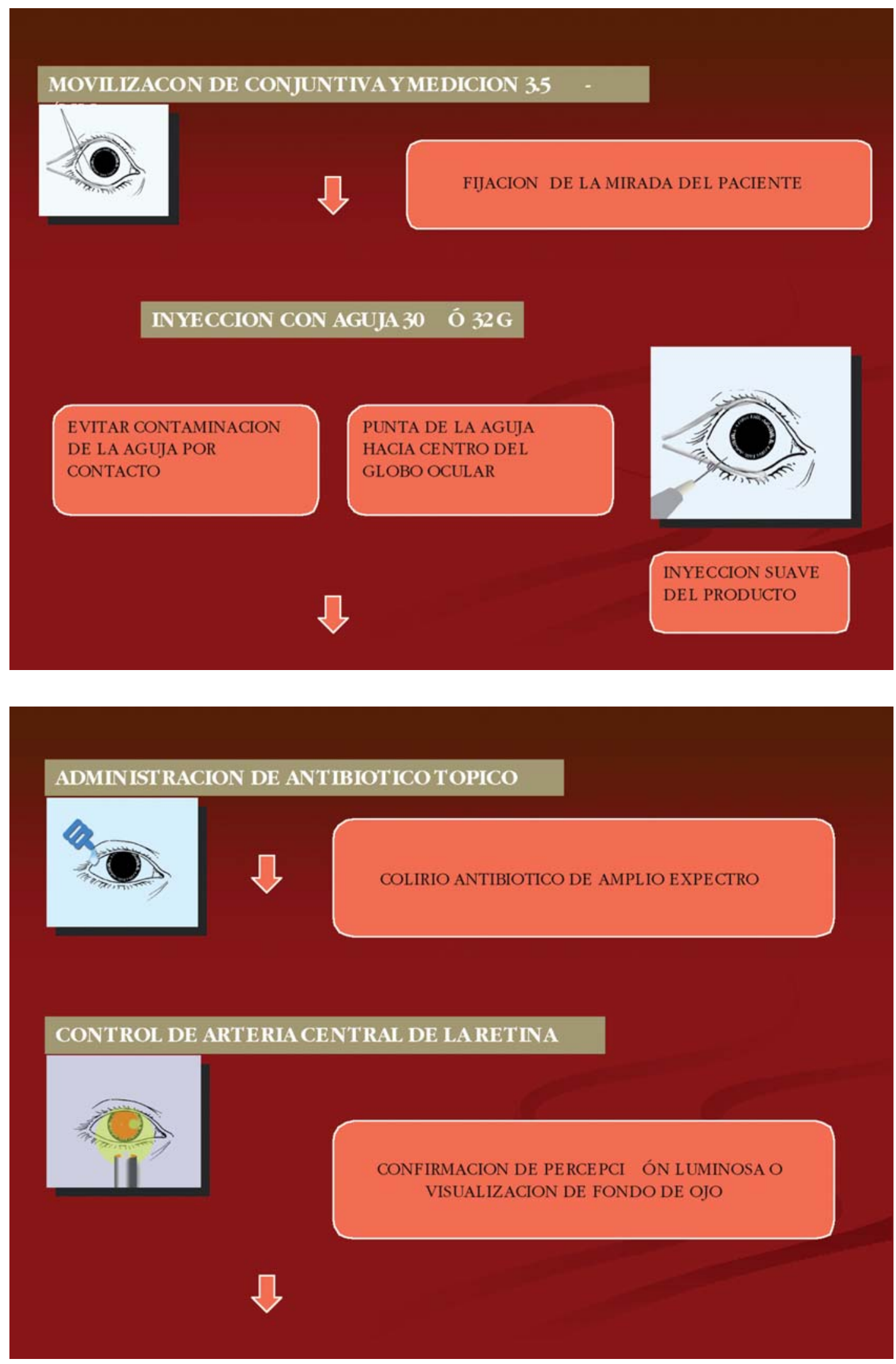


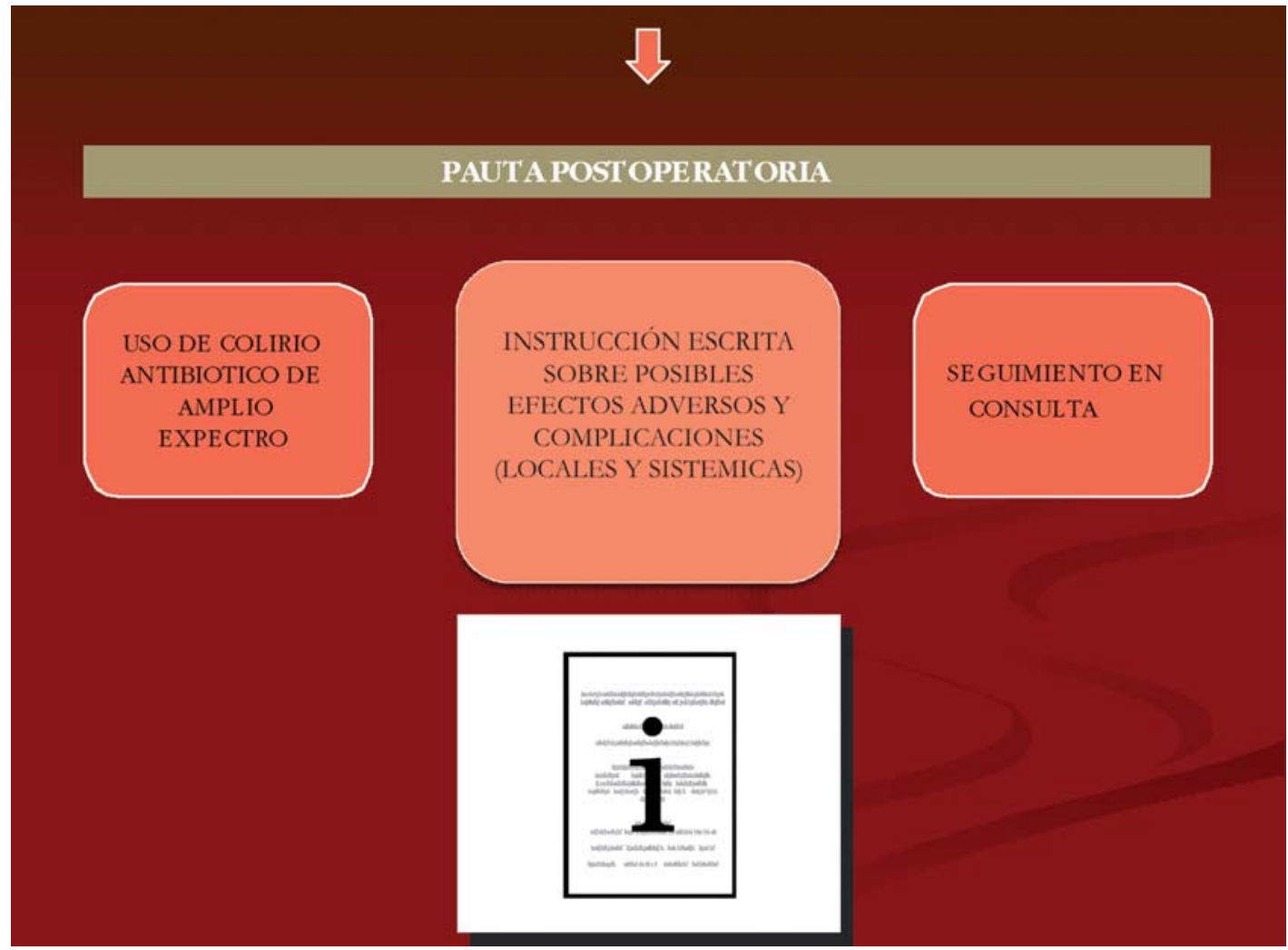

\section{BIBLIOGRAFÍA}

1. Ohm J. Über die Behandlung der Netzhautablo“sung durch operative Entleerung der subretinalen Flössigkeit und Einspritzung von Luft in den Glaskörper. Albrecht von Graefes Arch Ophthalmol 1911; 79: 442-450.

2. Rycroft B. Penicillin and the control of deep intraocular infection. Br J Ophthalmol 1945; 29: 57-87.

3. Schneider J, Frankel SS. Treatment of late postoperative intraocular infections with intraocular injection of penicillin. Arch Ophthalmol 1947; 37: 304-307.

4. Rosengren B. 300 cases operated upon for retinal detachment; method and results. Acta Ophthalmol (Copenh) 1952; 30: 117-122.

5. Cibis PA, Becker B, Okun E, Canaan S. The use of liquid silicone in retinal detachment surgery. Arch Ophthalmol 1962; 68: 590-599.

6. Domínguez Collazo A, Fonseca Sandomingo A, Gómez Montaña J. Cirugía precoz y ambulatoria del desprendimiento de retina. Arch Soc Esp Oftalmol 1985; 48: 47-54.

7. Dominguez A. Pneumocasis. Madrid: Ed. IDIOSA; 1995.

8. Forster RK, Zachary IG, Cottingham AJ Jr, Norton EW. Further observations on the diagnosis, cause, and treatment of endophthalmitis. Am J Ophthalmol 1976; 81: 52-56.

9. Peyman GA, Vastine DW, Raichand M. Experimental aspects and their clinical application. Ophthalmology 1978; 85: 374-385.

10. Henry K, Cantrill H, Fletcher C, Chinnock BJ, Balfour HH Jr. Use of intravitreal ganciclovir (dihydroxy propoxy- methyl guanine) for cytomegalovirus retinitis in a patient with AIDS. Am J Ophthalmol 1987; 103: 17-23.

11. Vitravene injection (fomivirsen sodium intravitreal injectable). Approval letter, pages 1-4. Vol 2004. US Food and Drug Administration. Disponible en: www.fda.gov/cder/foil nda/98/20961_Vitravene_Approv.pdf. Consultado el 29 de Abril de 2004.

12. Dominguez, A, Quiroga P, Jareño M. El tratamiento de enfermedades médicas con triamcinolona intravítrea. Arch Soc Esp Oftalmol 1993; 65: 491-498.

13. Domínguez. A. Dispositivos y medicamentos introducidos intraocularmente para el tratamiento en el consultorio de enfermedades de los ojos. An Real Acad Nac Med 1994; 61: 377-385.

14. Martidis A, Duker JS, Greenberg PB, Rogers AH, Puliafito $C A$, Reichel $E$, et al. Intravitreal triamcinolone for refractory diabetic macular edema. Ophthalmology 2002; 109: 920-927.

15. Greenberg PB, Martidis A, Rogers AH, Duker JS, Reichel $E$. Intravitreal triamcinolone acetonide for macular oedema due to central retinal vein occlusion. Br J Ophthalmol 2002; 86: 247-248.

16. Antcliff RJ, Spalton DJ, Stanford MR, Graham EM, Ffytche TJ, Marshall J. Intravitreal triamcinolone for uveitic cystoid macular edema: an optical coherence tomography study. Ophthalmology 2001; 108: 765-772.

17. Young S, Larkin G, Branley M, Lightman S. Safety and efficacy of intravitreal triamcinolone for cystoid macular oedema in uveitis. Clin Exp Ophthalmol 2001; 29: 2-6. 
18. Martidis A, Duker JS, Puliafito CA. Intravitreal triamcinolone for retinochoroidopathy. Arch Ophthalmol 2001; 119: 1380-1383.

19. Scott IU, Flynn HW Jr, Rosenfeld PJ. Intravitreal triamcinolone acetonide for idiopathic cystoid macular edema. Am J Ophthalmol 2003; 136: 737-739.

20. Gómez-Ulla F. Marticorena J, Alfaro DV 3rd, Fernández $M$, Méndez ER, Rothen M. Intravitreal triamcinolone for the treatment of diabetic macular edema. Curr Diabetes Rev 2006; 2: 99-112.

21. The Eyetech Study Group. Preclinical and phase 1A clinical evaluation of an anti-VEGF pegylated aptamer (EYE001) for the treatment of exudative age-related macular degeneration. Retina 2002; 22: 143-152.

22. The Eyetech Study Group. Anti-vascular endothelial growth factor therapy for subfoveal choroidal neovascularization secondary to age-related macular degeneration: phase II study results. Ophthalmology 2003; 110: 979-986.

23. Gragoudas ES, Adamis AP, Cunningham ET Jr, Feinsod M, Guyer DR; VEGF Inhibition Study in Ocular Neovascularization Clinical Trial Group. Pegaptanib for neovascular age-related macular degeneration. $N$ Engl J Med 2004; 351: 2805-2816.

24. Cunningham ET Jr, Adamis AP, Altaweel M, Aiello LP, Bressler NM, D'Amico DJ, et al. A phase II randomized double-masked trial of pegaptanib, an antivascular endothelial growth factor aptamer, for diabetic macular edema. Ophthalmology 2005; 112: 1747-1757.

25. Rosenfeld PJ, Brown DM, Heier JS, Boyer DS, Kaiser PK, Chung CY, et al. Ranibizumab for neovascular age-related macular degeneration. N Engl J Med 2006; 355: 14191431.

26. Brown DM, Kaiser PK, Michels M, Soubrane G, Heier JS, Kim RY, et al. Ranibizumab versus verteporfin for neovascular age-related macular degeneration. $N$ Engl J Med 2006; 335: 1432-1444.

27. Rosenfeld PJ, Brown DM, Heier JS, Boyer DS, Kaiser PK, Chung CY, et al. Ranibizumab for neovascular age-related macular degeneration. N Engl J Med 2006; 355: 14191431.

28. Dhalla MS, Shah GK, Blinder KJ, Ryan EH Jr, Mittra RA, Tewari A. Combined photodynamic therapy with verteporfin and intravitreal bevacizumab for choroidal neovascularization in age-related macular degeneration. Retina 2006; 26: 988-993.

29. Yoganathan P, Deramo VA, Lai JC, Tibrewala RK, Fastenberg DM. Visual improvement following intravitreal bevacizumab (avastin) in exudative age-related macular degeneration. Retina 2006; 26: 994-998.

30. Haritoglou C, Kook D, Neubauer A, Wolf A, Priglinger S, Strauss $R$, et al. Intravitreal bevacizumab (avastin) therapy for persistent diffuse diabetic macular edema. Retina 2006; 26: 999-1005.

31. Jorge R, Costa RA, Calucci D, Cintra LP, Scott IU. Intravitreal bevacizumab (avastin) for persistent new vessels in diabetic retinopathy (IBEPE Study). Retina 2006; 26: 1006-1013.

32. Rosenfeld PJ, Moshfeghi AA, Puliafito CA. Optical coherence tomography findings after an intravitreal injection of bevacizumab (Avastin) for neovascular age-related macular degeneration. Ophthalmic Surg Lasers Imaging 2005; 36: 331-335.
33. Jager RD, Aiello LP, Patel SC, Cunningham ET Jr. Risks of intravitreous injection: a comprehensive review. Retina 2004; 24: 676-698.

34. Fung AE, Rosenfeld PJ, Reichel E. The International Bevacizumab safety Surrey: using the Internet to assess drug safety Worldwide. Br J Ophthalmol 2006; 90: 1344-1349.

35. Wu L, Martínez-Castellanos MA, Quiroz-Mercado H, Arevalo JF, Berrocal MH, Farah ME, et al. Twelve-month safety of intravitreal injections of bevacizumab (Avastin): results of the Pan-American Collaborative Retina Study Group (PACORES). Graefes Arch Clin Exp Ophthalmol 2008; 246: 81-87.

36. Moshfeghi AA. Rate of endophthalmitis after Anti-VGEF after intravitreal injection. Retina Today March/April 2008; 75-76.

37. Pilli S, Kotsolis A, Spaide RF, Slakter J, Freund KB, Sorenson $J$, et al. Endophthalmitis associated with intravitreal anti-vascular endothelial growth factor therapy injections in an office setting. Am J Ophthalmol 2008; 145: 879-882.

38. Quiroz-Mercado H, Ustariz-González O, Martinez-Castellanos M, Covarrubias P, Dominguez, F, Sanchez-Huerta $V$. Our Experience after 1765 intravitreal injections of bevacizumab: the importance of being part of a developing story. Semin Ophthalmol 2007; 22: 109-125.

39. Heier JS, Antoszyk AN, Pavan PR, Leff SR, Rosenfeld PJ, Ciulla TA, et al. Ranibizumab for treatment of neovascular age-related macular degeneration: a phase I/II multicenter, controlled, multidose study. Ophthalmology 2006; 113: 633-642.

40. Westfall AC, Osborn A, Kuhl D, Benz, MS, Mieler WF, Holz, ER. Acute endophthalmitis incidence. Intravitreal triamcinolone. Arch Ophthalmol 2005; 123: 1075-1077.

41. Moshfeghi DM, Kaiser PK, Scott IU, Sears JE, Benz M, Sinesterra JP, et al. Acute endophthalmitis following intravitreal triamcinolone acetonide injection. Am J Ophthalmol 2003; 136: 791-796.

42. Jonisch J, Lai JC, Deramo VA, Flug AJ, Fastenberg DM. Increased incidence of sterile endophtahlmitis following itravitreal preserved triamcinolone. $\mathrm{Br} J$ Ophthalmol 2008; 92: 1051-1054.

43. Moshfeghi AA, Scott IU, Flynn HW Jr, Puliafito CA. Pseudohypopyon after intravitreal triamcinolone acetonide injection for cystoid macular edema. Am J Ophthalmol 2004; 138: 489-492.

44. Moshfeghi DM, Kaiser PK, Bakri SJ, Kaiser RS, Maturi $R K$, Sears JE, et al. Presumed sterile endophthalmitis following intravitreal triamcinolone acetonide injection. Ophthalmic Surg Lasers Imaging 2005; 36: 24-29.

45. Aiello LP, Brucker AJ, Chang S, Cunningham ET Jr, Damico DJ, Flynn HW Jr, et al. Evolving guidelines for intravitreal injections. Retina 2004; 24: S3-S19.

46. Jaissle GB, Szurman P, Bartz-Schmidt KU. Empfehlung fur die Durcgfuhrung von intravitrealen Injektionen. Klin Monatsbl Augenheilkd 2005; 222: 390-395.

47. The Royal College of Ophthalmologists. Intravitrel injections Procedure Guideline». (www.rcophth.ac.uk/docs/z-old-website-documents/IVT_Inject_Guidelines_FINAL_for_website.pdf).

48. Speaker MG, Milch FA, Shah MK, Eisner W, Kreiswirth $B N$. Role of external bacterial flora in the pathogenesis of acute postoperative endophthalmitis. Ophthalmology 1991; 98: 639-650. 
49. Bannerman TL, Rhoden DL, McAllister SK, Miller JM, Wilson LA. The source of coagulase-negative staphylococci in the Endophthalmitis Vitrectomy Study. A comparison of eyelid and intraocular isolates using pulsed-field gel electrophoresis. Arch Ophthalmol 1997; 115: 357-361.

50. Han DP, Wisniewski SR, Wilson LA, Barza M, Vine AK, Doft BH, et al. Spectrum and susceptibilities of microbiologic isolates in the Endophthalmitis Vitrectomy Study. Am J Ophthalmol 1996; 122: 1-17.

51. Leong JK, Shah R, McCluskey PJ, Benn RA, Taylor RF. Bacterial contamination of the anterior chamber during phacoemulsification cataract surgery. $J$ Cataract Refract Surg 2002; 28: 826-833.

52. Hovding G, Sjursen H. Bacterial contamination of drops and dropper tips of in-use multidose eye drop bottles. Acta Ophthalmol (Copenh) 1982; 60: 213-222.

53. Kozak I, Cheng L, Freeman WR. Lidocaine gel anesthesia for intravitreal drug administration. Retina 2005; 25: 994 998.

54. Miller JJ, Scott IU, Flynn HW Jr, Smiddy WE, Newton J, Miller D. Acute-onset endophthalmitis after cataract surger y (2000-2004): incidence, clinical settings, and visual acuity outcomes after treatment. Am J Ophthalmol 2005; 139: 983-987.

55. Ferguson AW, Scott JA, McGavigan J. Comparison of 5\% povidone iodine solution against $1 \%$ povidone-iodine solution in preoperative cataract surgery antisepsis: a prospective randomised double blind study. Br J Ophthalmol 2003; 87: 163-167.

56. Speaker MG, Menikoff JA. Prophylaxis of endophthalmitis with topical povidone-iodine. Ophthalmology 1991; 98: 1769-1775.

57. Miño de Kaspar H, Chang RT, Singh K, Egbert PR, Blumenkranz MS, Ta CN. Prospective Randomized Comparison of 2 Different Methods of 5\% Povidone-Iodine Appli- cations for Anterior Segment Intraocular Surgery. Arch Ophthalmol 2005; 123: 161-165.

58. Isenberg S, Apt L, Yoshimori R, Khwarg S. Chemical preparation of the eye in ophthalmic surgery $I V$ : comparison of povidone-iodine on the conjunctiva with a prophylactic antibiotic. Arch Ophthalmol 1985; 103: 1340-1342.

59. Grimes S, Mein C, Trevino S. Preoperative antibiotic and povidone-iodine preparation of the eye. Ann Ophthalmol 1991; 23: 263-266.

60. Snyder-Perimutter L,Katz HR, Melia M. Effect of topical ciprofloxacin $0.3 \%$ and ofloxacin $0.3 \%$ on the reduction of bacterial flora on the human conjunctiva. J Cataract Refract Surg 2000; 26: 1620-1625.

61. Ta CN, Egbert PR, Singh K, Shriver EM, Blumenkranz MS, Miño De Kaspar H. Prospective randomized comparison of 3-day versus 1-hour preoperative ofloxacin prophylaxis for cataract surgery. Ophthalmology 2002; 109: 2036-2041.

62. Osher RH, Amdahl LD, Cheetham JK. Antimicrobial efficacy and aqueous humor concentration of preoperative and postoperative topical trimethoprim/polymyxin B sulfate versus tobramycin. J Cataract Refract Surg 1994; 20: 3-8.

63. Deramo VA, Lai JC, Fastenberg DM, Udell IJ. Acute endophthalmitis in eyes treated prophylactically with gatifloxacin and moxifloxacin. Am J Ophthalmol 2006; 142: 721-725.

64. Miller D, Flynn PM, Scott IU, Alfonso EC, Flynn HW Jr. In vitro fluoroquinolone resistance in staphylococcal endophthalmitis isolates. Arch Ophthalmol 2006; 124: 479-483.

65. Bucci FA, Amico LM, Guerino A, Evans R. The effect of preop lid scrubs and levofloxacin on eyelid and conjunctival cultures prior to cataract surgery. Invest Ophthalmol Vis Sci 2003; 44: E-Abstract 1446-B342. 